\title{
WEAK CONVERGENCE AND WEAK COMPACTNESS FOR MULTIFUNCTIONS WITH VALUES IN A SEPARABLE BANACH SPACE
}

\author{
NIKOLAOS S. PAPAGEORGIOU
}

(Communicated by R. Daniel Mauldin)

\begin{abstract}
In this paper we extend the notion of weak convergence to Banach space valued integrable multifunctions. We prove some properties of this mode of convergence and we show that under certain hypotheses the space of uniformly bounded, $w$-compact, convex valued integrable multifunctions is sequentially weakly complete. Then we prove two Dunford-Pettis type weak compactness theorems and we also show that the set valued conditional expectation is weakly continuous. Finally we present an application from control theory.
\end{abstract}

\section{INTRODUCTION}

In the last twenty years the subject of measurable multifunctions has been developed extensively and has found important applications in various fields, like mathematical economics, optimal control and optimization.

In this paper we study weakly convergent sequences of Banach space valued multifunctions. The notion of weak convergence of multifunctions was first introduced by Artstein [1] for $\mathbf{R}^{n}$-valued multifunctions and later extended to multifunctions with values in a separable reflexive Banach space by this author [13, section 4]. Here we improve the results of [13] and obtain new ones. First we derive various alternative characterizations of this type of set convergence. Then we present a completeness result, two Dunford-Pettis type compactness theorems and we also show that the set valued conditional expectation is weakly continuous. Finally we present an application from control theory. Some of the above results extend earlier ones by the author, as we will explain in detail in the sequel. See Theorems 4.1 and 4.3 in [13] and Theorem 4.1 in [14].

Received by the editors February 2, 1989 and, in revised form, September 11, 1989.

1980 Mathematics Subject Classification (1985 Revision). Primary 28A45, 46G10.

Key words and phrases. Integrably bounded multifunction, multimeasure, Dunford-Pettis theorem, multivalued Radon-Nikodym theorem, Mackey topology, set valued conditional expectation, mild solution, control system.

Research supported by NSF Grant DMS- 8802688 . 
Let $(\Omega, \Sigma, \mu)$ be a complete, finite measure space and $X$ a separable Banach space. We will be using the following notations:

$$
P_{f(c)}(X)=\{A \subseteq X: \text { nonempty, closed, (convex) }\}
$$

and

$$
P_{(w) k(c)}(X)=\{A \subseteq X: \text { nonempty, }(w-) \text { compact, (convex) }\} .
$$

A multifunction (set valued functions) $F: \Omega \rightarrow P_{f}(X)$, is said to be measurable, if for all $z \in X, \omega \rightarrow d(z, F(\omega))=\inf \{\|z-x\|: x \in F(\omega)\}$ is measurable. By $S_{F}^{1}$ we will denote the set of all Bochner integrable selectors of $F(\cdot)$; i.e., $S_{F}^{1}=\left\{f \in L^{1}(X): f(\omega) \in F(\omega) \mu\right.$-a.e. $\}$. It is easy to see using Aumann's selection theorem (see Wagner [20]), that $S_{F}^{1}$ is nonempty if and only if $\omega \rightarrow \inf \{\|x\|: x \in F(\omega)\}$ belongs in $L_{+}^{1}$. A multifunction $F(\cdot)$ is said to be "integrably bounded," if it is measurable and $\omega \rightarrow\{\|x\|: x \in F(\omega)\}$ belongs in $L_{+}^{1}$. Clearly for such a multifunction $S_{F}^{1}$ is nonempty. Using $S_{F}^{1}$ we can define a set valued integral for $F(\cdot)$, by setting $\int_{\Omega} F(\omega) d \mu(\omega)=\left\{\int_{\Omega} f(\omega) d \mu(\omega): f \in\right.$ $\left.S_{F}^{1}\right\}$.

For $A \in 2^{X} \backslash\{\varnothing\}$, we define the function $\sigma_{A}: X^{*} \rightarrow \mathbf{R}=\mathbf{R} \cup\{+\infty\}$ by $\sigma_{A}\left(x^{*}\right)=\sup \left\{\left(x^{*}, x\right): x \in A\right\}$. This is known as the "support function" of the set $A$. A multifunction $M: \Sigma \rightarrow P_{w k c}(X)$ is said to be a "multimeasure" (set valued measure), if for all $x^{*} \in X^{*}, A \rightarrow \sigma_{M(A)}\left(x^{*}\right)$ is a signed measure.

Finally the set valued conditional expectation of a measurable multifunction $F: \Omega \rightarrow P_{f(c)}(X)$ with respect to a sub- $\sigma$-field $\Sigma_{0}$ of $\Sigma$, is defined to be the measurable multifunction $E^{\Sigma_{0}} F: \Omega \rightarrow P_{f(c)}(X)$ such that $S_{E^{\Sigma_{0}}}^{1}=\overline{E^{\Sigma_{0}} S_{F}^{1}}$, the closure taken in $L^{1}(X)$. If $F(\cdot)$ is integrably bounded, then clearly so is $E^{\Sigma_{0}} F(\cdot)$. For details we refer to Hiai-Umegaki [9].

\section{WEAK CONVERGENCE}

In [1], Artstein extended the notion of weak convergence for $L^{1}$-functions to $\mathbf{R}^{n}$-valued multifunctions. Artstein's work was extended by this author [13] to multifunctions with values in a separable, reflexive Banach space. Here we strengthen the results of [13] and present new ones.

Recall that by the Dinculeanu-Foias theorem (see Ionescu-Tulcea [10]), we have that $L^{1}(X)^{*}=L^{\infty}\left(X_{u w^{*}}^{*}\right)$. If $F_{n}, F: \Omega \rightarrow P_{f c}(X)$ are integrably bounded multifunctions, then we say that the $F_{n}$ 's converge weakly to $F$, denoted by $F_{n} \stackrel{w}{\rightarrow} F$, if for all $x^{*}(\cdot) \in L^{\infty}\left(X_{w^{*}}^{*}\right)$,

$$
\int_{\Omega} \sigma_{F_{n}(\omega)}\left(x^{*}(\omega)\right) d \mu(\omega) \rightarrow \int_{\Omega} \sigma_{F(\omega)}\left(x^{*}(\omega)\right) d \mu(\omega) \text {. }
$$

Recall (see Hiai-Umegaki [9] or [16]) that $\int_{\Omega} \sigma_{F_{n}(\omega)}\left(x^{*}(\omega)\right) d \mu(\omega)=\sigma_{S_{F_{n}^{\prime}}}\left(x^{*}\right)$ and $\int_{\Omega} \sigma_{F(\omega)}\left(x^{*}(\omega)\right) d \mu(\omega)=\sigma_{S_{F}^{1}}\left(x^{*}\right)$. Also note that because $F(\cdot)$ is convex valued, the weak limit is unique. 
Theorem 2.1. If $F_{n}, F: \Omega \rightarrow P_{f c}(X)$ are integrably bounded multifunctions, then $F_{n} \stackrel{w}{\rightarrow} F$ if and only if, for all $z^{*} \in X^{*}, \sigma_{F_{n}(\cdot)}\left(z^{*}\right) \stackrel{w}{\rightarrow} \sigma_{F(\cdot)}\left(z^{*}\right)$ in $L^{1}(\mathbf{R})$.

Proof.

Necessity. First note that since $F_{n}, F$ are measurable we can find measurable functions $\left\{f_{n}^{m}\right\}_{m \geq 1}$ and $\left\{f^{m}\right\}_{m \geq 1}$ from $\Omega$ into $X$ such that $F_{n}(\omega)=$ $\left\{\overline{f_{n}^{m}(\omega)}\right\}_{m \geq 1}$ and $F(\omega)=\left\{\overline{f^{m}(\omega)}\right\}_{m \geq 1}$. See Wagner [20]. So $\omega \rightarrow \sigma_{F_{n}(\omega)}\left(z^{*}\right)=$ $\sup _{m \geq 1}\left(z^{*}, f_{n}^{m}(\omega)\right)$ and $\omega \rightarrow \sigma_{F(\omega)}\left(z^{*}\right)=\sup _{m \geq 1}\left(z^{*}, f^{m}(\omega)\right)$ are measurable and also integrable since $F_{n}, F$ are integrably bounded. Now let $x^{*}(\cdot)=$ $\chi_{A}(\cdot) z^{*}$ with $A \in \Sigma$. Then we have $\int_{A} \sigma_{F_{n}(\omega)}\left(z^{*}\right) d \mu(\omega)=\int_{\Omega} \sigma_{F_{n}(\omega)}\left(x^{*}(\omega)\right) d \mu(\omega)$ $\rightarrow \int_{\Omega} \sigma_{F(\omega)}\left(x^{*}(\omega)\right) d \mu(\omega)=\int_{A} \sigma_{F(\omega)}\left(z^{*}\right) d \mu(\omega)$. Since $A \in \Sigma$ was arbitrary, we conclude that $\sigma_{F_{n}(\cdot)}\left(z^{*}\right) \stackrel{w}{\rightarrow} \sigma_{F(\cdot)}\left(z^{*}\right)$ in $L^{1}$.

Sufficiency. Let $x^{*}(\cdot) \in L^{\infty}\left(X_{w^{*}}^{*}\right)$ be a simple function; i.e., $x^{*}(\omega)=$ $\sum_{k=1}^{n} z_{k}^{*} \chi_{A_{k}}(\omega)$, with $z_{k}^{*} \in X^{*}, A_{k} \in \Sigma$. Then we have

$$
\begin{aligned}
\sigma_{S_{F_{n}}}\left(x^{*}\right) & =\int_{\Omega} \sigma_{F_{n}(\omega)}\left(x^{*}(\omega)\right) d \mu(\omega) \\
& =\sum_{k=1}^{n} \int_{A_{k}} \sigma_{F(\omega)}\left(z_{k}^{*}\right) d \mu(\omega) \\
& \rightarrow \sum_{k=1}^{n} \int_{A} \sigma_{F(\omega)}\left(z_{k}^{*}\right) d \mu(\omega) \\
& =\int_{\Omega} \sigma_{F(\omega)}\left(x^{*}(\omega)\right) d \mu(\omega)=\sigma_{S_{F}^{1}}\left(x^{*}\right) .
\end{aligned}
$$

Now recall that any $x^{*}(\cdot) \in L^{\infty}\left(X_{u^{*}}^{*}\right)$ can be approximated in the Mackey topology $m\left(L^{\infty}\left(X_{w^{*}}^{*}\right), L^{1}(X)\right)$ by simple functions. Also note that since $S_{F_{n}}^{1}, S_{F}^{1} \in$ $P_{u k c}\left(L^{1}(X)\right)$ [12, Proposition 3.1], we have that $\sigma_{S_{F_{n}}^{1}}(\cdot), \sigma_{S_{F}^{1}}(\cdot)$ are $m$-continuous. So by a standard density argument we conclude that $\sigma_{S_{F_{n}}^{1}}\left(x^{*}\right) \rightarrow \sigma_{S_{F}^{1}}\left(x^{*}\right)$ for all $x^{*}(\cdot) \in L^{\infty}\left(X_{w^{*}}^{*}\right) \Rightarrow F_{n} \stackrel{w}{\rightarrow} F$. Q.E.D.

Another characterization of the weak convergence is provided by the next theorem. This theorem was first proved by the author in [13, theorem 4.1], under the additional hypotheses that $\mu(\cdot)$ is nonatomic, $X$ is reflexive, and $\left|F_{n}(\omega)\right|=\sup \left\{\|x\|: x \in F_{n}(\omega)\right\},|F(\omega)|=\sup \{\|x\|: x \in F(\omega)\} \leq h(\omega) \mu$-a.e. with $h(\cdot) \in L_{+}^{1}$. Here we drop all these extra hypotheses.

Let $x^{*}(\cdot) \in L^{\infty}\left(X_{w^{*}}^{*}\right)$, and set

$$
\begin{aligned}
L_{n}\left(x^{*}\right)(\omega) & =\left\{\left(x^{*}(\omega), x\right): x \in F_{n}(\omega)\right\}, \\
L\left(x^{*}\right)(\omega) & =\left\{\left(x^{*}(\omega), x\right): x \in F(\omega)\right\} .
\end{aligned}
$$

Also by $h(\cdot, \cdot)$ we will denote the Hausdorff metric on $P_{k c}(\mathbf{R})$; i.e., for $A, B \in$ $P_{k c}(\mathbf{R}), h(A, B)=\max \left(\sup _{a \in A} d(a, B), \sup _{b \in B} d(b, A)\right)$. 
Theorem 2.2. If $F_{n}, F: \Omega \rightarrow P_{w k c}(X)$ are integrably bounded multifunctions then $F_{n} \stackrel{w}{\rightarrow} F$ if and only if, for all $x^{*}(\cdot) \in L^{\infty}\left(X_{w^{*}}^{*}\right), \int_{\Omega} L_{n}\left(x^{*}\right) d \mu(\omega) \stackrel{h}{\rightarrow}$ $\int_{\Omega} L\left(x^{*}\right)(\omega) d \mu(\omega)$.

Proof.

Necessity. Note that $L_{n}\left(x^{*}\right)(\omega), L\left(x^{*}\right)(\omega) \in P_{k c}(\mathbf{R})$. Also if

$$
\left\{f_{n}^{m}(\cdot)\right\}_{m \geq 1},\left\{f^{m}(\cdot)\right\}_{m \geq 1}
$$

are measurable functions from $\Omega$ into $X$ such that $F_{n}(\omega)=\left\{\overline{f_{n}^{m}(\omega)}\right\}_{m \geq 1}$ and $F(\omega)=\left\{f^{m}(\omega)\right\}_{m \geq 1}($ see Wagner [20]), then

$$
L_{n}\left(x^{*}\right)(\omega)=\left\{\left(x^{*}(\omega), f_{n}^{m}(\omega)\right)\right\}_{m \geq 1}
$$

and $L\left(x^{*}\right)(\omega)=\left\{\left(x^{*}(\omega), f^{m}(\omega)\right)\right\}_{m>1}$, which shows that $L_{n}\left(x^{*}\right)(\cdot), L\left(x^{*}\right)(\cdot)$ are measurable and moreover integrably bounded for all $x^{*}(\cdot) \in L^{\infty}\left(X_{w^{*}}^{*}\right)$. Let $p \in \mathbf{R}$. We have $\int_{\Omega} \sigma(p)_{L_{n}\left(x^{*}\right)(\omega)} d \mu(\omega)=\int_{\Omega}$ p. $\sigma_{F_{n}(\omega)}\left(x^{*}(\omega)\right) d \mu(\omega) \rightarrow \int_{\Omega}$ p. $\sigma_{F(\omega)}\left(x^{*}(\omega)\right) d \mu(\omega)=\int_{\Omega}^{n} \sigma(p)_{L\left(x^{*}\right)(\omega)} d \mu(\omega)$. But recall (see for example Artstein [1] and Papageorgiou [15]) that $\int_{\Omega} \sigma(p)_{L_{n}\left(x^{*}\right)(\omega)} d \mu(\omega)=\sigma_{\int_{\Omega}} L_{n}\left(x^{*}\right)(p)$

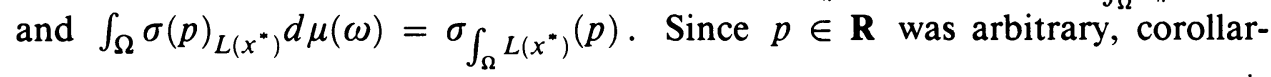
ies P4A and 3A of Salinetti-Wets [18], tell us that $\int_{\Omega} L_{n}\left(x^{*}\right)(\omega) d \mu(\omega) \stackrel{h}{\rightarrow}$ $\int_{\Omega} L\left(x^{*}\right)(\omega) d \mu(\omega)$.

Sufficiency. Note that $\int_{\Omega}$ p. $\sigma_{F_{n}(\omega)}\left(x^{*}(\omega)\right) d \mu(\omega)=\int_{\Omega} \sigma_{L_{n}\left(x^{*}\right)(\omega)}(p) d \mu(\omega)$ and $\int_{\Omega}$ p. $\sigma_{F(\omega)}\left(x^{*}(\omega)\right) d \mu(\omega)=\int_{\Omega}^{n} \sigma(p)_{L\left(x^{*}\right)(\omega)} d \mu(\omega)$, while

$$
\int_{\Omega} \sigma(p)_{L_{n}\left(x^{*}\right)(\omega)} d \mu(\omega) \rightarrow \int_{\Omega} \sigma_{L\left(x^{*}\right)(\omega)}(p) d \mu(\omega) .
$$

Take $p=1$. Then the sufficiency part follows. Q.E.D.

\section{WEAK COMPLETENESS}

We will say that a sequence $\left\{F_{n}\right\}_{n \geq 1}$ and $P_{f c}(X)$-valued integrably bounded multifunctions is "weakly Cauchy" if and only if for all $x^{*}(\cdot) \in L^{\infty}\left(X_{w^{*}}^{*}\right)$,

$$
\left\{\int_{\Omega} \sigma_{F_{n}(\omega)}\left(x^{*}(\omega)\right) d \mu(\omega)\right\}_{n \geq 1}
$$

is Cauchy in $L^{1}$. The next result shows that under certain hypotheses, a weakly Cauchy sequence converges to an integrably bounded multifunction with the same kind of values.

Theorem 3.1. If both $X$ and $X^{*}$ have the Radon-Nikodym property (RNP), $F_{n}: \Omega \rightarrow P_{w k c}(X)$ are measurable multifunctions such that $F_{n}(\omega) \subseteq W(\omega) \mu$ a.e. with $W: \Omega \rightarrow P_{w k c}(X)$ integrably bounded and $\left\{F_{n}\right\}_{n \geq 1}$ is weakly Cauchy, then there exists $F: \Omega \rightarrow P_{w k c}(X)$ measurable multifunction such that $F(\omega) \subseteq$ $W(\omega)$ u-a.e. and $F_{n} \stackrel{w}{\rightarrow} F$. 
Proof. By considering if necessary $\overline{\operatorname{conv}}[W(\omega) \cup(-W(\omega))]$, which by the KreinSmulian theorem (see Diestel-Uhl [6], Theorem 11, p. 51) is still in $P_{w k c}(X)$, we may assume without any loss of generality that for all $\omega \in \Omega, W(\omega)$ is symmetric. Let $A \in \Sigma$ and $z^{*} \in X^{*}$. Then from Proposition 2.1 of [15], we have $\sigma_{\int_{A} F_{n}}\left(z^{*}\right)=\int_{A} \sigma_{F_{n}(\omega)}\left(z^{*}\right) d \mu(\omega)$ and this by hypothesis converges to a limit that in general depends on $\left(A, z^{*}\right)$. Denote that limit by $\phi\left(A, z^{*}\right)$. Clearly $\phi(A, \cdot)$ is sublinear and since $W(\cdot)$ is assumed to have symmetric values we have $\left|\phi\left(A, z^{*}\right)\right| \leq \sigma_{\int_{A} W}\left(z^{*}\right)$. From the corollary to Proposition 3.1 in [12], we know that $\int_{A} W \in P_{w k c}(X)$. So $\sigma_{\int_{A} W}(\cdot)$ is $m\left(X^{*}, X\right)$-continuous (where $m\left(X^{*}, X\right)$ denotes the Mackey topology on $X^{*}$ for the pair $\left(X^{*}, X\right)$ ). Thus for every $A \in \Sigma$, there exists $M(A) \in P_{w k c}(X)$ such that $\phi\left(A, z^{*}\right)=$ $\sigma_{M(A)}\left(z^{*}\right)$. From Nikodym's theorem, we know that $A \rightarrow \phi\left(A, z^{*}\right)=\sigma_{M(A)}\left(z^{*}\right)$ is a signed measure; thus $M(\cdot)$ is a multimeasure. Invoking Theorem 3 of Costé [3], we get that there exists $F: \Omega \rightarrow P_{w k c}(X)$ integrably bounded such that $M(A)=\int_{A} F(\omega) d \mu(\omega)$. So, for all $A \in \Sigma$ and $z^{*} \in X^{*}, \int_{\dot{A}} \sigma_{F_{n}(\omega)}\left(z^{*}\right) d \mu(\omega) \rightarrow$ $\int_{A} \sigma_{F(\omega)}\left(z^{*}\right) d \mu(\omega)$ and this by Theorem 2.1 tells us that $F_{n} \stackrel{{ }^{\prime}}{\rightarrow} F$. Q.E.D.

\section{Weak compactness Results of the Dunford-PetTis type}

In this section we present two "weak compactness" results of the DunfordPettis type for $P_{w k c}(X)$-valued multifunctions.

From Corollary 8 of Diestel-Uhl [6, p. 198 ], we know that if $X$ is separable, then $X^{*}$ has the RNP if and only if it is separable.

Theorem 4.1. If both $X$ and $X^{*}$ have the $R N P, F_{n}: \Omega \rightarrow P_{w k c}(X)$ are measurable multifunctions such that $F_{n}(\omega) \subseteq W(\omega)$ u-a.e. with $W: \Omega \rightarrow P_{u k c}(X)$ integrably bounded, then there exists a measurable multifunction $F: \Omega \rightarrow P_{u k c}(X)$ with $F(\omega) \subseteq W(\omega)$-a.e. and a subsequence $\left\{F_{n_{k}}\right\}_{k \geq 1}$ of $\left\{F_{n}\right\}_{n \geq 1}$ such that $F_{n_{k}} \stackrel{w}{\rightarrow} F$.

Proof. As before we can assume without loss of generality that $W(\cdot)$ has symmetric values. Let $D_{0}^{*}$ be a countable dense subset of $X^{*}$ and let $D^{*}$ be the countable dense subset of $X^{*}$ obtained from $D_{0}^{*}$ by taking rational linear combinations of its elements; i.e., $D^{*}=\left\{\sum_{k=1}^{n} \lambda_{k} z_{k}^{*}: n \geq 1, \lambda_{k}=\right.$ rational, $z_{k}^{*} \in$ $\left.D_{0}^{*}\right\}$. Enumerate the elements of $D^{*}$, i.e., $D^{*}=\left\{z_{m}^{*}\right\}_{m \geq 1}$. By the classical Dunford-Pettis compactness criterion, we know that $\left\{\sigma_{F_{n}(\cdot)}\left(z_{1}^{*}\right)\right\}_{n \geq 1}$ is relatively sequentially $w$-compact in $L^{1}$. So by passing to a subsequence (denoted for notational simplicity by the same index as the initial sequence), we get, for all $A \in \Sigma, \int_{A} \sigma_{F_{n}(\omega)}\left(z_{1}^{*}\right) d \mu(\omega)=\sigma_{\int_{A}^{r_{n}}}\left(z_{1}^{*}\right) \rightarrow \phi\left(A, z_{1}^{*}\right)$. A new application of the Dunford-Pettis theorem gives us a further subsequence (again denoted by the same index) such that $\int_{A} \sigma_{F_{n}(\omega)}\left(z_{2}^{*}\right) d \mu(\omega)=\sigma_{\int_{A} F_{n}}\left(z_{2}^{*}\right) \rightarrow \phi\left(A, z_{2}^{*}\right)$. We continue this way deriving sub-sub-subsequences. Then by a standard diagonal 
process, we get a final subsequence of the original sequence such that, for all $z^{*} \in D^{*}, \int_{A} \sigma_{F_{n}(\omega)}\left(z^{*}\right) d \mu(\omega)=\sigma_{\int_{A} F_{n}}\left(z^{*}\right) \rightarrow \phi\left(A, z^{*}\right)$.

Note that because of the sublinearity of the support function and since $\int_{A} W$ is symmetric, we have for any $z^{*}, z^{* \prime} \in D^{*}:\left|\sigma_{\int_{A} F_{n}}\left(z^{*}\right)-\sigma_{\int_{A} F_{n}}\left(z^{* \prime}\right)\right| \leq$ $\sigma_{\int_{A} W}\left(z^{*}-z^{* \prime}\right)$. Passing to the limit as $n \rightarrow \infty$, we get $\left|\phi\left(A, z^{*}\right)-\phi\left(A, z^{* \prime}\right)\right| \leq$ $\sigma_{\int_{A} W}\left(z^{*}-z^{* \prime}\right) \leq\left|\int_{A} W\right| \cdot\left\|z^{*}-z^{* \prime}\right\|$. From this inequality we deduce that $\phi(A, \cdot)$ is uniformly strongly continuous on $D^{*}$, which is strongly dense in $X^{*}$. So there exists a unique uniformly strongly continuous extension $\hat{\phi}(A, \cdot)$ of $\phi(A, \cdot)$ on $X^{*}$. By a straightforward limit argument as $n \rightarrow \infty$ and since $D^{*}=\left\{\right.$ Rational linear combinations of elements in $\left.D_{0}^{*}\right\}$, we have

$$
\phi\left(A, z^{*}+z^{* \prime}\right) \leq \phi\left(A, z^{*}\right)+\phi\left(A, z^{* \prime}\right) \quad \text { for all } z^{*}, z^{* \prime} \in D^{*}
$$

and $\lambda \phi\left(A, z^{*}\right)=\phi\left(A, \lambda z^{*}\right), \lambda=$ Rational, $z^{*} \in D^{*}$.

Then by a density argument we conclude that $\hat{\phi}(A, \cdot)$ is sublinear. Also observe that $\left|\phi\left(A, z^{*}\right)\right| \leq \sigma_{\int_{A} W}\left(z^{*}\right)$ for all $z^{*} \in X^{*}$ and recall $\int_{A} W \in P_{w k c}(X)$ (see [12]). So $\hat{\phi}(A, \cdot)$ is $m\left(X^{*}, X\right)$-continuous. Thus there exists $M(A) \in$ $P_{w k c}(X)$ such that $\phi\left(A, z^{*}\right)=\sigma_{M(A)}\left(z^{*}\right)$ for all $z^{*} \in X^{*}$.

Let $z^{*} \in X^{*}$. Then we can find $\left\{z_{m_{k}}^{*}\right\}_{k \geq 1}=\left\{z_{k}^{*}\right\}_{k \geq 1}$ a subsequence of $D^{*}$ such that $z_{k}^{*} \stackrel{s}{\rightarrow} z^{*}$. We have:

$$
\sigma_{\int_{A} F_{n}}\left(z_{k}^{*}\right) \rightarrow \phi\left(A, z_{k}^{*}\right) \text { as } n \rightarrow \infty
$$

and $\hat{\phi}\left(A, z_{k}^{*}\right)=\phi\left(A, z_{k}^{*}\right) \rightarrow \hat{\phi}\left(A, z^{*}\right)$ as $k \rightarrow \infty$.

By diagonalization we can find a subsequence $k(n)$ such that $\sigma_{\int_{A} F_{n}}\left(z_{k(n)}^{*}\right) \rightarrow$ $\phi\left(A, z^{*}\right)$ as $n \rightarrow \infty$. Using this fact, we have for any $\left(A, z^{*}\right) \in \Sigma \times X^{*}$ : $\left|\sigma_{\int_{A} F_{n}}\left(z^{*}\right) \rightarrow \hat{\phi}\left(A, z^{*}\right)\right| \leq\left|\sigma_{\int_{A} F_{n}}\left(z^{*}\right)-\sigma_{\int_{A} F_{n}}\left(z_{k(n)}^{*}\right)\right|+\left|\sigma_{\int_{A} F_{n}}\left(z_{k(n)}^{*}\right)-\hat{\phi}\left(A, z^{*}\right)\right|$. Note that $\left|\sigma_{\int_{A} F_{n}}\left(z^{*}\right)-\sigma_{\int_{A} F_{n}}\left(z_{k(n)}^{*}\right)\right| \leq \sigma_{\int_{A} W}\left(z-z_{k(n)}^{*}\right) \rightarrow 0$ as $n \rightarrow \infty$. Also from the choice of the subsequence $k(n)$, we have $\left|\sigma_{\int_{A} F_{n}}\left(z_{k(n)}^{*}\right)-\hat{\phi}\left(A, z^{*}\right)\right| \rightarrow 0$ as $n \rightarrow \infty$. Thus finally we have $\sigma_{\int_{A} F_{n}}\left(z^{*}\right) \rightarrow \hat{\phi}\left(A, z^{*}\right)=\sigma_{M(A)}\left(z^{*}\right)$ and by Nikodym's theorem we deduce that $A \rightarrow \sigma_{M(A)}\left(z^{*}\right)$ is a signed measure, which implies that $M(\cdot)$ is a multimeasure. Apply Theorem 3 of Costé [3], to get $F: \Omega \rightarrow P_{w k c}(X)$ integrably bounded such that $F(\omega) \subseteq W(\omega) \mu$-a.e. and $M(A)=\int_{A} F(\omega) d \mu(\omega)$ for all $A \in \Sigma$. Then for every $\left(A, z^{*}\right) \in \Sigma \times X^{*}$, we have $\sigma_{\int_{A} F_{n}}\left(z^{*}\right)=\int_{A} \sigma_{F_{n}(\omega)}\left(z^{*}\right) d \mu(\omega) \rightarrow \sigma_{\int_{A} F}\left(z^{*}\right)=\int_{A} \sigma_{F(\omega)}\left(z^{*}\right) d \mu(\omega) \Rightarrow$ $\sigma_{F_{n}(\cdot)}\left(z^{*}\right) \stackrel{w}{\rightarrow} \sigma_{F(\cdot)}\left(z^{*}\right)$ in $L^{1}(\mathbf{R})$ and so by Theorem 2.1 , we finally have $F_{n} \stackrel{w}{\rightarrow}$ $F$. Q.E.D.

By strengthening our boundedness hypothesis on $\left\{F_{n}\right\}_{n \geq 1}$ we can drop the RNP-hypothesis on $X^{*}$ (or equivalently the separability hypothesis on $X^{*}$ ). This theorem was actually proved in [14, Theorem 4.1], without using the notion 
of weak convergence as is defined here. Here we state the conclusion of the theorem using the notion of weak convergence introduced in this paper and we also present a second proof, which in fact shows that theorem 4.2 (and so theorem 4.1 of [14] too), is a special case of theorem 4.1.

Theorem 4.2. If $F_{n}: \Omega \rightarrow P_{w k c}(X)$ are measurable multifunctions such that $F_{n}(\omega) \subseteq W$ u-a.e. with $W \in P_{w k c}(X)$, then there exists $F: \Omega \rightarrow P_{w k c}(X)$ measurable multifunction such that $F(\omega) \subseteq W$ M-a.e. and a subsequence $\left\{F_{n_{k}}\right\}_{k \geq 1}$ of $\left\{F_{n}\right\}_{n \geq 1}$ such that $F_{n_{k}} \stackrel{w}{\rightarrow} F$ as $k \rightarrow \infty$.

First proof. This proof was first presented in [14, Theorem 4.1] and is reproduced here for completeness. It follows the steps of the proof of theorem 4.1, with some appropriate modifications. We will only indicate those modifications.

In this case $D^{*}$ is going to be $m\left(X^{*}, X\right)$-dense (see Willansky [21, p. 144]). As in the proof of Theorem 4.1, by a diagonal process, we get a subsequence of the original sequence (denoted for simplicity by the same index) such that for all $\left(A, z^{*}\right) \in \Sigma \times D^{*} \sigma_{\int_{A} F_{n}}\left(z^{*}\right) \rightarrow \phi\left(A, z^{*}\right)$. Also for all $z^{*}, z^{* \prime} \in D^{*}$ we have $\left|\phi\left(A, z^{*}\right)-\phi\left(A, z^{* \prime}\right)\right| \leq \sigma_{\int_{A} W}\left(z^{*}-z^{* \prime}\right)$, which shows that $\phi(A, \cdot)$ is continuous on $D^{*}$. Now note that if $\hat{z}^{*} \in X^{*}$ and $\left\{z_{\alpha}^{*}\right\}_{\alpha \in \Gamma}$ and $\left\{z_{\beta}^{*}\right\}_{\beta \in \Gamma}$ are nets in $D^{*}$ indexed by the Mackey filterbase $\mathscr{U}_{m}\left(\hat{z}^{*}\right)$ of $\hat{z}^{*}$, we have $\mid \phi\left(A, z_{\alpha}^{*}\right)-$ $\phi\left(A, z_{\beta}^{*}\right) \mid \rightarrow 0$; i.e., $\left\{\phi\left(A, z_{\alpha}^{*}\right)\right\}_{\alpha \in \Gamma}$ and $\left\{\phi\left(A, z_{\beta}^{*}\right)\right\}_{\sigma \in \Gamma}$ have the same limit. Hence we can apply Theorem $5.3,[7$, p. 216], to get a unique $m$-continuous extension $\hat{\phi}(A, \cdot)$ of $\phi(A, \cdot)$ on $X^{*}$. As in the proof of Theorem 4.1, we can check that $\phi(A, \cdot)$ is sublinear and $\hat{\phi}\left(A, z^{*}\right)=\sigma_{M(A)}\left(z^{*}\right)$ with $M(A) \in$ $P_{w k c}(X)$.

Let $\hat{z}^{*} \in X^{*}$ and $z^{*} \in D^{*}$. We have:

$$
\begin{aligned}
\left|\sigma_{\int_{A} F_{n}}\left(\hat{z}^{*}\right)-\sigma_{M(A)}\left(\hat{z}^{*}\right)\right| & \\
\leq & \left|\sigma_{\int_{A} F_{n}}\left(\hat{z}^{*}\right)-\sigma_{\int_{A} F_{n}}\left(z^{*}\right)\right|+\left|\sigma_{\int_{A} F_{n}}\left(z^{*}\right)-\sigma_{M(A)}\left(z^{*}\right)\right| \\
& \quad+\left|\sigma_{M(A)}\left(z^{*}\right)-\sigma_{M(A)}\left(\hat{z}^{*}\right)\right| \\
& \leq 2 \mu(A) \sigma_{W}\left(\hat{z}^{*}-z^{*}\right)+\left|\sigma_{\int_{A} F_{n}}\left(z^{*}\right)-\sigma_{M(A)}\left(z^{*}\right)\right| .
\end{aligned}
$$

Choose $z^{*} \in D^{*}$ such that $\sigma_{W}\left(\hat{z}^{*}-z^{*}\right)<\varepsilon / 4 \mu(A)$. This is possible since $D^{*}$ is $m$-dense in $X^{*}$ and $\sigma_{W}(\cdot)$ is $m$-continuous (because $\left.W \in P_{w k c}(X)\right)$. Also let $n_{0} \geq 1$ be such that, for $n \geq n_{0},\left|\sigma_{\int_{A} F_{n}}\left(z^{*}\right)-\sigma_{M(A)}\left(z^{*}\right)\right|<\varepsilon / 2$. Thus finally for $n \geq n_{0}$ we have $\left|\sigma_{\int_{A} F_{n}}\left(\hat{z}^{*}\right)-\sigma_{M(A)}\left(\hat{z}^{*}\right)\right|<\varepsilon \Rightarrow \sigma_{\int_{A} F_{n}}\left(\hat{z}^{*}\right) \rightarrow \sigma_{M(A)}\left(\hat{z}^{*}\right)$ as $n \rightarrow \infty$ for all $\left(A, \hat{z}^{*}\right) \in \Sigma \times X^{*}$. Hence by Nikodym's theorem $\sigma_{M(\cdot)}\left(\hat{z}^{*}\right)$ is a signed measure, thus $M(\cdot)$ is a multimeasure. Now apply the set valued RadonNikodym theorem of Godet-Thobie [8], to get $F: \Omega \rightarrow P_{w k c}(X)$ integrably bounded such that $F(\omega) \subseteq W$-a.e. and $M(A)=\int_{A} F(\omega) d \mu(\omega)$. Then for 
all $\left(A, \hat{z}^{*}\right) \in \Sigma \times X^{*}$, we have $\int_{A} \sigma_{F_{n}(\omega)}\left(\hat{z}^{*}\right) d \mu(\omega)=\sigma_{\int_{A} F_{n}}\left(z^{*}\right) \rightarrow \sigma_{\int_{A} F}\left(z^{*}\right) \rightarrow$ $\sigma_{\int_{A} F}\left(z^{*}\right)=\int_{A} \sigma_{F(\omega)}\left(z^{*}\right) d \mu(\omega)$, which by Theorem 2.1 implies that $F_{n} \stackrel{w}{\rightarrow} F$.

Second proof. As we already said, we may assume that $W$ is absolutely convex (i.e., symmetric) and weakly compact. Then from a result of Davis-FigelJohnson-Pelczynski [4] (see also Diestel [5, p. 163]), we know that $W$ can be obtained as the one-to-one weakly bicontinuous image of the unit ball of a separable, reflexive Banach space $Y \stackrel{j}{\hookrightarrow} X$; i.e., $W=j(C)$, where $C$ is the unit ball of $Y$ and $j(\cdot)$ is an affine homeomorphism in the weak topology. Set $\hat{F}(\omega)=j^{-1}(F(\omega))$. Recall that $F(\omega)={\left.\overline{\left\{f_{n}(\omega)\right.}\right\}_{n \geq 1}^{w}}_{\text {where } f_{n}: \Omega \rightarrow X n \geq 1}$ are measurable. Then $\hat{F}(\omega)={\overline{\left\{j^{-1}\left(f_{n}(\omega)\right)\right.}}_{n \geq 1}^{w}$ and $j^{-1} \circ f_{n}(\cdot) n \geq 1$ are weakly measurable, hence measurable, because $Y$ is separable (Pettis's measurability theorem, see Diestel-Uhl [6, p. 42]). So we may work with $\left\{\hat{F}_{n}(\cdot)\right\}_{n \geq 1}$ in $Y$. Recalling that $Y^{*}$ is reflexive too, and that reflexive Banach spaces have the RNP, then Theorem 4.2 follows as a special case of Theorem 4.1. Q.E.D.

Remark. We could have used this approach in the proof of Theorem 4.1. But then we would have to deal with a parameterized family of separable reflexive Banach spaces and this does not simplify the proof.

\section{Set valued conditional eXpectation}

Our next result shows that the set valued conditional expectation is continuous with respect to the weak convergence. An analogous result was proved earlier by the author [13, Theorem 4.3], but under stronger hypotheses. Namely there $X$ was separable, reflexive and for $\left|F_{n}(\omega)\right|=\sup \left\{\|x\|: x \in F_{n}(\omega)\right\}$ and $|F(\omega)|=\sup \{\|x\|: x \in F(\omega)\}$, we had $\left|F_{n}(\omega)\right|,|F(\omega)| \leq h(\omega) \quad \mu$-a.e. with $h(\cdot) \in L_{+}^{1}$. As we will see, here we weaken considerably these hypotheses. The notion of set valued conditional expectation turned out to be useful in the study of generalized information systems (see deKorvin-Kleyle [11]), in optimization and optimal control (see Bismut [2]), and in mathematical economics (see Papageorgiou [16]).

Theorem 5.1. If $X^{*}$ is separable, $F_{n}, F: \Omega \rightarrow P_{f c}(X)$ are integrably bounded multifunctions, $F_{n} \stackrel{w}{\rightarrow} F$ and $\Sigma_{0}$ is a sub- $\sigma$-field of $\Sigma$, then $E^{\Sigma_{0}} F_{n} \stackrel{w}{\rightarrow} E^{\Sigma_{0}} F$ as $n \rightarrow \infty$.

Proof. Note that since $X^{*}$ is separable it has the RNP and so by Theorem 1, $\left[6\right.$, p. 98], $L^{\infty}\left(\Sigma_{0}, X^{*}\right)=\left[L^{1}\left(\Sigma_{0}, X\right)\right]^{*}$. Let $x^{*}(\cdot) \in L^{\infty}\left(\Sigma_{0}, X^{*}\right)$. Then from Thibault [19], we know that

$$
\int_{\Omega} \sigma_{F_{n}(\omega)}\left(x^{*}(\omega)\right) d \mu(\omega)=\int_{\Omega} E^{\Sigma_{0}} \sigma_{F_{n}(\omega)}\left(x^{*}(\omega)\right) d \mu(\omega) .
$$

Also from Lemma 2.1 of [17], we have that $E^{\Sigma_{0}} \sigma_{F_{n}(\omega)}\left(x^{*}(\omega)\right)=\sigma_{E^{\Sigma_{0}} F_{n}(\omega)}\left(x^{*}(\omega)\right)$ $\mu$-a.e. Thus we have $\int_{\Omega} \sigma_{F_{n}(\omega)}\left(x^{*}(\omega)\right) d \mu(\omega)-\int_{\Omega}^{n} \sigma_{E^{\Sigma_{0}} F_{n}(\omega)}\left(x^{*}(\omega)\right) d \mu(\omega)$. Sim- 
ilarly $\int_{\Omega} \sigma_{F(\omega)}\left(x^{*}(\omega)\right) d \mu(\omega)=\int_{\Omega} \sigma_{E^{\Sigma_{0}} F(\omega)}\left(x^{*}(\omega)\right) d \mu(\omega)$. Since $F_{n} \stackrel{w}{\rightarrow} F$ we have

$$
\begin{aligned}
\int_{\Omega} \sigma_{F_{n}(\omega)}\left(x^{*}(\omega)\right) d \mu(\omega) & \rightarrow \int_{\Omega} \sigma_{F(\omega)}\left(x^{*}(\omega)\right) d \mu(\omega) \\
& \Rightarrow \int_{\Omega} \sigma_{E} \Sigma_{F_{F_{n}}(\omega)}\left(x^{*}(\omega)\right) d \mu(\omega) \rightarrow \int_{\Omega} \sigma_{E^{\Sigma_{0}(\omega)}}\left(x^{*}(\omega)\right) d \mu(\omega) \\
& \Rightarrow E^{\Sigma_{0_{F_{n}}} \stackrel{w}{\rightarrow} E^{\Sigma_{0_{F}}} \text { as } n \rightarrow \infty .}
\end{aligned}
$$

Q.E.D.

\section{AN EXAMPLE}

In this section we use the theory of weak convergence of multifunctions to study the dependence of the attainable (reachable) set $R(t)$ of an infinite dimensional linear control system on the control constraint set.

Let $T=[0, b], X$ a separable Banach space (modeling the state space) and $Y$ another separable Banach space (modeling the control space). The control system under consideration is the following:

$$
\left\{\begin{array}{l}
\dot{x}(t)=A(t) x(t)+B(t) u(t) \\
x(0)=x_{0}, u(t) \in U(t) \text { a.e. }
\end{array}\right\}(*) .
$$

Here $\{A(t)\}_{t \in T}$ is a family of generally unbounded linear operators defined on $D(A(t))=D \subseteq X$ and generating an evolution operator $S:\{(t, s): 0 \leq s \leq$ $t \leq b\} \rightarrow \mathscr{L}(X)$, which is compact for $t-s>0$. Also $B \in L^{\infty}(T, \mathscr{L}(Y, X))$. By a solution of $(*)$, we understand a mild solution $x_{u}(\cdot) \in C(T, X)$; i.e., $x_{u}(t)=S(t, 0) x_{0}+\int_{0}^{t} S(t, s) B(s) u(s) d s$ for some $u(\cdot) \in S_{U}^{1}$.

Let $U_{n}, U: T \rightarrow P_{f c}(Y)$ be measurable multifunctions such that $\left|U_{n}(t)\right|=$ $\sup \left\{\|u\|: u \in U_{n}(t)\right\}$ and $|U(t)|=\sup \{\|u\|: u \in U(t)\}$ are both less or equal to $\phi(t)$ a.e. where $\phi(\cdot) \in L_{+}^{1}$. We define $R_{n}(t)=\left\{x_{u}(t): x_{u}(\cdot)\right.$ is a mild solution of $(*)$ with $\left.u(\cdot) \in S_{U_{n}}^{1}\right\}$ and $R(t)=\left\{x_{u}(t): x_{u}(\cdot)\right.$ is a mild solution of $(*)$ with $\left.u(\cdot) \in S_{U}^{1}\right\}$; i.e., these are the attainable sets of $(*)$ with admissible controls in $S_{U_{n}}^{1}$ and $S_{U}^{1}$ respectively. Let $h(\cdot, \cdot)$ denote the Hausdorff metric on $P_{k c}(X)$.

Theorem 6.1. If the above hypotheses hold and $U_{n} \stackrel{w}{\rightarrow} U$, then $R_{n}(t) \stackrel{h}{\rightarrow} R(t)$ as $n \rightarrow \infty$.

Proof. Let $W(t)=\{u \in Y:\|u\| \leq \phi(t)\}$ and set

$$
V(t)=S(t, 0) x_{0}+\int_{0}^{t} S(t, s) B(s) W(s) d s .
$$

Since the hypothesis $S(t, s)$ is compact for $t-s>0$, from Radström's embedding theorem (see Hiai-Umegaki [9]), we deduce that for all $t \in T, V(t) \in$ $P_{k c}(X)$. Then by Mazur's theorem (see Diestel-Uhl [6], Theorem 12, p. 51), $\hat{V}(t)=\overline{\operatorname{conv}}(V(t) \cup(-V(t))) \in P_{k c}(X)$. Also note that $\left\{R_{n}(t), R(t)\right\} \subseteq P_{k c}(X)$ for all $t \in T$. So for all $t \in T$, from the Banach-Dieudonne theorem we 
have that $\left\{\sigma_{R_{n}(t)}(\cdot), \sigma_{R(t)}(\cdot), \sigma_{\hat{V}(t)}(\cdot)\right\}_{n \geq 1}$ are sequentially $w^{*}$-continuous. Let $B_{1}^{*}=\left\{z^{*} \in X^{*}:\left\|z^{*}\right\| \leq 1\right\}$ (the closed unit ball in $X^{*}$ ). This with the relative $w^{*}$-topology is a compact, metrizable space. For $z^{*}, z^{* \prime} \in B_{1}^{*}$ we have $\left|\sigma_{R_{n}(t)}\left(z^{*}\right)-\sigma_{R_{n}(t)}\left(z^{* \prime}\right)\right| \leq \sigma_{\hat{V}(t)}\left(z^{*}-z^{* \prime}\right)$ for all $n \geq 1 \Rightarrow\left\{\sigma_{R_{n}(t)}(\cdot)\right\}_{n \geq 1}$ is an equicontinuous subset of $C\left(B_{1}^{*}, w^{*}\right)$. Also it is clearly bounded. Hence applying the Arzela-Ascoli theorem, we deduce that by passing to a subsequence if necessary, we may assume that $\left\{\sigma_{R_{n}(t)}(\cdot)\right\}_{n \geq 1}$ converges uniformly on $B_{1}^{*}$.

On the other hand note that for every $z^{*} \in X^{*}$ we have:

$$
\sigma_{R_{n}(t)}\left(z^{*}\right)=\left(S(t, 0) x_{0}, z^{*}\right)+\int_{0}^{t} \sigma_{U_{n}(s)}\left(B^{*}(s) S(t, s)^{*} z^{*}\right) d s
$$

and $\sigma_{R(t)}\left(z^{*}\right)=\left(S(t, 0) x_{0}, z^{*}\right)+\int_{0}^{t} \sigma_{U(s)}\left(B^{*}(s) S(t, s)^{*} z^{*}\right) d s, t \in T$.

Since $U_{n} \stackrel{w}{\rightarrow} U$, we have $\sigma_{R_{n}(t)}\left(z^{*}\right) \rightarrow \sigma_{R(t)}\left(z^{*}\right) \Rightarrow \sigma_{R_{n}(t)}(\cdot) \rightarrow \sigma_{R(t)}(\cdot)$ uniformly on $B_{1}^{*} \Rightarrow R_{n}(t) \stackrel{h}{\rightarrow} R(t)$ for all $t \in T$. Q.E.D.

Remark. If $U_{n}(t)=\left\{u_{n}(t)\right\} n \geq 1$ and $U(t)=\{u(t)\}$, then the above theorem tells us that the trajectories vary continuously with weakly convergent controls; i.e., $u_{n} \stackrel{w}{\rightarrow} u \Rightarrow x_{u_{n}}(t) \stackrel{s}{\rightarrow} x_{u}(t)$. In fact it is easy to check that the convergence is uniform in $t \in T$; i.e., $x_{u_{n}}(\cdot) \stackrel{s}{\rightarrow} x_{u}(\cdot)$ in $C(T, X)$.

\section{ACKNOWLEDGMENT}

The author is grateful to the referee for his constructive criticism and suggestions that helped improve the material included in this paper.

\section{REFERENCES}

1. Z. Artstein, Weak convergence of set valued functions and control, Siam J. Control 13 (1975), 865-878.

2. J.-M. Bismut, Integrales convexes et probabilités, J. Math. Anal. Appl. 42 (1973), 639-673.

3. A. Costé, La propriété de Radon-Nikodym en integration multivoque, C. R. Acad. Sci. Paris 280 (1975), $1515-1518$.

4. W. Davis, T. Figiel, W. Johnson, and A. Pelczynski, Factoring weakly compact operators, J. Funct. Anal. 17 (1974), 311-327.

5. J. Diestel, Geometry on Banach spaces-selected topics, Lecture Notes in Math., vol. 465, Springer, Berlin, 1975.

6. J. Diestel and J. Uhl, Vector Measures, Math. Surveys, 15, Amer. Math. Soc., Providence, RI, 1977.

7. J. Dugundji, Topology, Allyn and Bacon, Boston, MA, 1966.

8. C. Godet-Thobie, Theoremes de Radon-Nikodym multivoque et integration par rapport a certaines multimesures, Sem. Anal. Convexe, Montpellier, 1974, exp. 11, pp. 11.1-11.9.

9. F. Hiai and H. Umegaki, Integrals, conditional expectations and martingales of multivalued functions, J. Multiv. Anal. 7 (1977), 149-182.

10. A. and C. Ionescu-Tulcea, Topics in the theory of lifting, Springer, Berlin, 1969.

11. A. de Korvin and R. Kleyle, A convergence theorem for convex set valued supermartingales, Stoch. Anal. Appl. 3 (1985), 433-445. 
12. N. S. Papageorgiou, On the theory of Banach space valued multifunctions. Part 1: Integration and conditional expectation, J. Multiv. Anal. 17 (1985), 185-206.

13. __ On the theory of Banach space valued multifunctions. Part 2: Set valued martingales and set valued measures, J. Multiv. Anal. 17 (1985), 207-227.

14. Contributions to the theory of set valued functions and set valued measures, Trans. Amer. Math. Soc. 304 (1987), 245-265.

15. __ A relaxation theorem for differential inclusions in Banach spaces, Tôhoku Math. J. 39 (1987), 505-517.

16. (1986), 452-479.

17. _ A convergence theorem for set valued supermartingales in a separable Banach space, Stoch. Anal. Appl. 5 (1987), 405-422.

18. G. Salinetti and R. Wets, On the convergence of sequences of convex sets in finite dimensions, SIAM Rev. 21 (1979), 18-33.

19. L. Thibault, Esperances conditionelles d'integrandes semi-continus, Ann. Inst. H. Poincaré XVII (1981), 337-350.

20. D. Wagner, Survey of measurable selection theorems, SIAM J. Control and Optim. 15 (1977), 859-903.

21. A. Willansky, Modern methods in topological vector spaces, McGraw-Hill, New York, 1978.

1015 Department of Mathematics, University of California, Davis, California 95616 